\title{
UHF Defect Evaluation in Gas Insulated Equipment
}

\author{
S. Meijer and J.J. Smit \\ Delft University of Technology \\ Faculty of Information Systems and Technology \\ Department of High Voltage Technology and Management \\ Mekelweg 4, 2628 CD Delft, The Netherlands
}

\begin{abstract}
Small defects in $\mathrm{SF}_{6}$ gas-insulated substations like fixed particles (fixed to conductors or on spacer surfaces) or free moving particles can influence the insulation strength of the insulation system. To determine the risk of such defects on the insulation strength, identification of the PD source is one of the most important steps. In this paper, a method to identify defects based on statistical analysis of frequency spectra is introduced. Finally two examples of risk analysis of defects in GIS are shown.
\end{abstract}

Index Terms - Gas insulation, $\mathbf{S F}_{6}$, diagnostics, partial discharges, UHF measurements.

\section{INTRODUCTION}

$\mathrm{S}^{\mathrm{I}}$ INCE the early seventies, $\mathrm{SF}_{6}$ gas-insulated substations (GISs) are in use all over the world. Initially it was claimed that GISs were maintenance-free, had less influence to environmental and industrial conditions and resulted in a reduction of substation space and increase of safety. As a result, many substations were installed at important nodes in the electricity grid. They are used for distribution of electricity, protection of the grid and transformers can be present to transform, when necessary, the voltage level to the desired level.

Although several failures over the last 30 years have been reported in two international surveys, GISs have proven to be very reliable [1]. However, a failure can involve high costs, which can mainly be divided into repair costs and costs for non-delivery of energy. Therefore, means to analyse the risk of insulation defects and methods to prevent failures are definitely needed. In particular, the occurrence of defects like free particles and protrusions fixed to the conductors can be very dangerous to the insulation conditions of the GIS [2]. Depending on the type of insulation defect, a breakdown can follow immediately, after days, months or even after years. This means that the detection and recognition of these defects at an early stage are important to avoid possible failure of GIS.

As known, a breakdown is often preceded by partial discharge (PD) activity [3]. In service, usually low PD activity occurs, which itself is not dangerous for the operation of a GIS. Moreover, it can be a good indication of the

Manuscript received on 10 April 2002, in final form 3 November 2003. condition of the insulation. Therefore, one of the most sensitive means to predict an upcoming failure involving one of these defects, is the detection of partial discharge activity [2].

For undesirable events in the insulation system of $\mathrm{SF}_{6}$ gas-insulated systems an analysis process to assess the risk (called risk analysis process) involves the following four steps in condition assessment [4]: detection, identification and location of the PD source and finally risk assessment of the defect. This paper mainly focuses on a method to identify defects based on combined statistical analysis of frequency spectra and phase-resolved PD patterns. Measurements have been performed using the narrow-band UHF method and the location of PD sources is determined by time-of-flight measurements [2]. Finally two examples of analysis of risk for breakdown of defects in GIS are shown.

\section{PARTIAL DISCHARGE DETECTION IN GIS}

To detect partial discharges in GIS the ultra-high frequency (UHF) method has been used [5-7]. To pick up the high-frequency electromagnetic waves excited somewhere inside the GIS, either internal or external UHF couplers can be used, (1a) and (1b) in Figure 1. A preamplifier (Figure 1 (2)) is directly connected to the coupler to amplify the picked up signals. A spectrum analyser (Figure 1 (3)) (SA, type HP 8590L) analyses the coupler's signals in the frequency domain, to obtain a frequency spectrum up to $1.8 \mathrm{GHz}$ and in the time domain, resulting in similar phase-resolved PD patterns that are obtained with a measuring circuit according to the IEC 60270 [8]. 


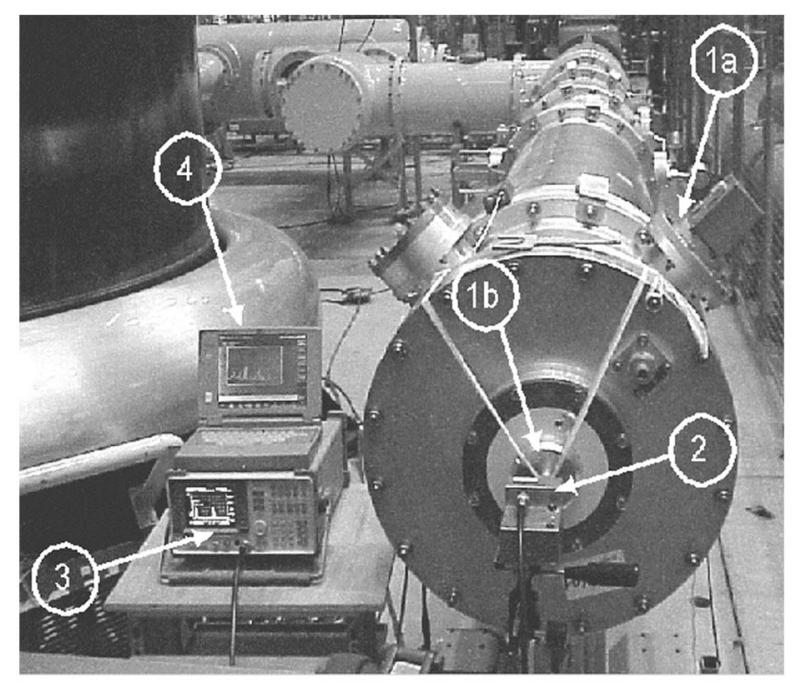

Figure 1. UHF measuring system. 1a, internal UHF coupler; 1b, external UHF coupler; 2, pre-amplifier ; 3, spectrum analyser; 4, PC.

A personal computer (Figure 1 (4)) has been used for further processing and analysis of the measuring results.

Since the magnitudes of the detected UHF signals depend on the location and type of the PD source, calibration of the PD magnitude as with a conventional measuring system is not possible. CIGRE Joint Working Group $33 / 23.12$ proposed test procedures for on-site testing of GIS, including partial discharge measurements with a maximum permissible PD of about $5 \mathrm{pC}$ [9]. Therefore, to guarantee also a sensitivity of at least $5 \mathrm{pC}$ with the UHF measuring circuit, a sensitivity check has been proposed by the CIGRÉ TF 15/33.03.05 [10]. However, PD identification methods based on phase-resolved or pulse sequence PD patterns do not need the amplitude to analyse the type of defect.

\subsection{MEASUREMENTS IN THE FREQUENCY DOMAIN}

The PD pulse shape (i.e. the rise and fall time, pulse duration and pulse height) and the time between subsequent pulses varies continuously during the PD process. As a result, peaks present in one sweep of the frequency spectrum can disappear in the next sweep. Therefore further analysis of a series of sweeps should reveal frequency ranges which occur in each sweep, using

- Hold max: derive a stable frequency spectrum from the maximum amplitude at each frequency as measured during the series of sweeps.

- Averaging: derive a stable frequency spectrum by averaging the measured amplitudes at each frequency over the series of sweeps.

As shown in [11], a measurement consisting of 20 sweeps of $5 \mathrm{~s}$ results in a post-processed frequency spectrum containing sufficient information, irrespective of the PD source type and the method of post-processing the frequency spectrum.

\subsection{MEASUREMENTS IN THE TIME DOMAIN}

For further analysis of the PD activity in time domain, a suitable center frequency is selected. An SA was used to demodulate the signals into the range of a few $\mathrm{MHz}$ in the following way:

- The center frequency $f_{c}$ of the SA is set to the selected measuring frequency,

- The measured span is set to zero: the zero span mode of the SA,

- The sweep time is set to $20 \mathrm{~ms}$ to obtain phase-resolved patterns correlated to the $50 \mathrm{~Hz}$ sine wave.

Methods for recognition and classification of phase-resolved PD patterns measured with a conventional measuring circuit have also been adapted to the UHF range [12].

\subsection{MEASURING RESULTS}

It is known that a relationship exists between the shape of phase-resolved PD patterns and the discharge source (type of defect) [13]. Each discharging defect with its geometry, location in insulation, dielectric properties and local field is characterized by a specific PD sequence. Analysis of these sequences has shown to be a good means to distinguish the different discharge sources for identification of defects [13, 14]. Moreover, also the frequency spectra generated by different types of defects in GIS are different, see figure 2. Therefore, a defect identification method is introduced using statistical analysis of frequency spectra.

\section{AMPLITUDE ANALYSIS OF FREQUENCY SPECTRA}

\subsection{DEFINITION OF SPECTRAL KEY VALUES}

In this section four spectral key values are proposed to collect information about the amplitudes of the measured signal. From a frequency spectrum which is built up from $\mathrm{N}$ data points $(\mathrm{N}=400$ in case of a HP $8590 \mathrm{~L})$, sampled during one sweep, these key values are processed in the following way.

- Measured power in the frequency spectrum (MP)

For the MP key value, all $\mathrm{N}$ measured amplitudes $\mathrm{S}_{\mathrm{i}}$ from the spectrum are quadratically summed and converted into $[\mathrm{dBm}]$. This key value is proportional to the measured power in the frequency spectrum [15]

$$
M P=10 \log \left(\sum_{i=1}^{N} S_{i}^{2}\right)
$$

- Average power in the frequency spectrum (AP).

The calculated average power is also a measure of power in the frequency spectrum, however after smoothening neighbouring amplitudes. All $\mathrm{N}$ measured amplitudes $S_{i}$ and $S_{i+1}$ are averaged, squared, summed, divided by the 

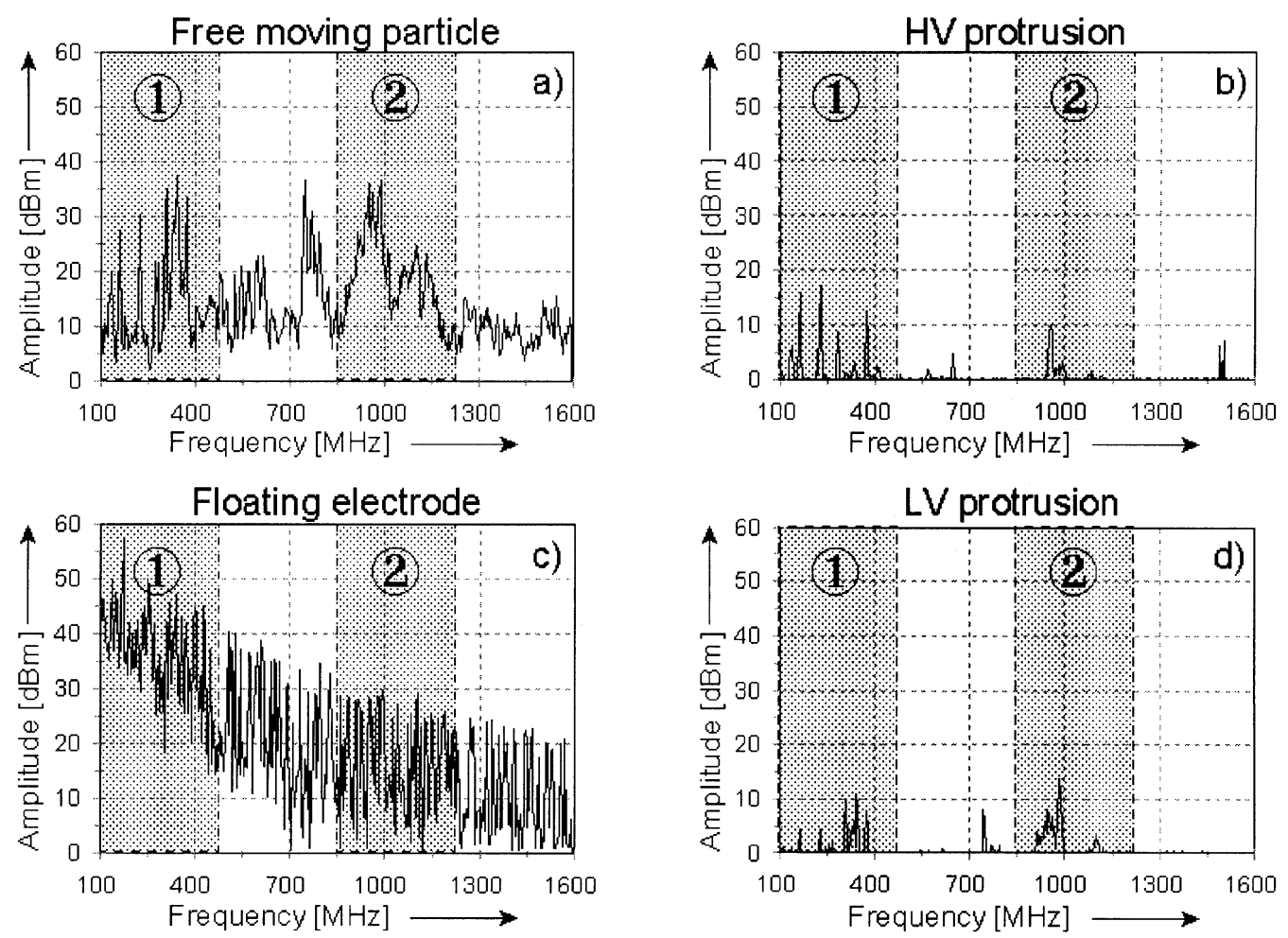

Figure 2. Signal-to-noise spectra measured for different defects using the same configuration. a, $15 \mathrm{~mm}$ free moving particle; $\mathrm{b}, 15 \mathrm{~mm}$ protrusion fixed to the conductor; c, electrically floating electrode at the conductor; $\mathrm{d}, 30 \mathrm{~mm}$ protrusion fixed to the enclosure.

number of data points and converted into $[\mathrm{dBm}]$

$$
A P=10 \log \left(\sum_{i=1}^{N}\left(\frac{S_{i}+S_{i+1}}{2}\right)^{2} \frac{1}{N}\right)
$$

- Maximum amplitude of the frequency spectrum (MA) A third key value is the maximum amplitude of the measured spectrum.

$$
M A=20 \log \left(\left.\operatorname{MAX}\left(S_{i}\right)\right|_{i=1} ^{N}\right)
$$

- Averaged area per data point (AR)

The spectra shown in Figure 2, are signal-to-noise frequency spectra. This means that the noise spectrum is subtracted from the signal spectrum. In this way, continuous noise signals coming from e.g. radio transmitters are suppressed. Based on these spectra the averaged area of these post-processed signal-to-noise-ratio frequency spectra can be calculated. All N processed amplitudes $S_{i}^{\prime}$ and $S_{i+1}$ are averaged, summed and divided by the number of data points

$$
A R=\sum_{i=1}^{N}\left(\frac{S_{i}^{\prime}+S_{i+1}^{\prime}}{2}\right) \frac{1}{N}
$$

These four key values can be calculated for the full measured frequency span or for a specific part of the frequency spectrum representing PD activity.

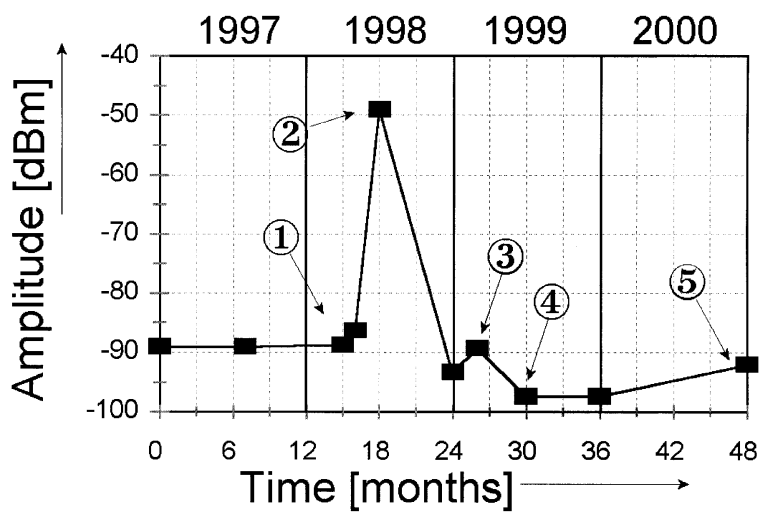

Figure 3. Trend analysis during a period of four years of key-value average power obtained at one of the UHF couplers in the $380 \mathrm{kV}$ GIS in Meeden, The Netherlands.

\subsection{TREND ANALYSIS OF PD SPECTRA OVER TIME}

The key-values are a representation of the power in the frequency spectrum. These values can be used to compare frequency spectra in an easy way. As an example, since 12 December 1996, UHF measurements have been performed in a $380 \mathrm{kV}$ substation in The Netherlands. The signals at this coupler have been monitored during ten measuring sessions over a time-period of four years. Figure 3 shows the key value AP (averaged power) processed from the obtained frequency spectra for all measuring 
a)
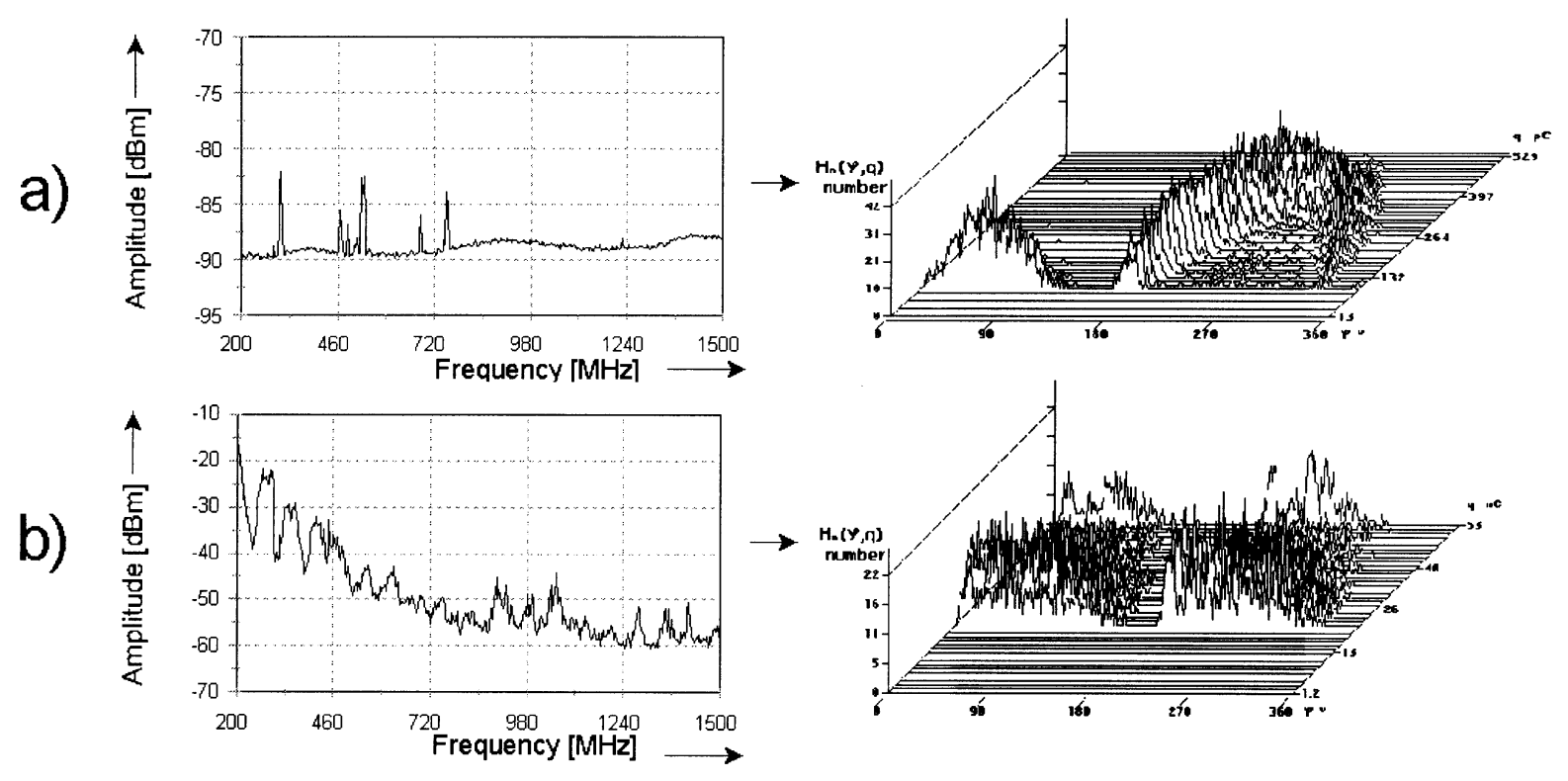

c)

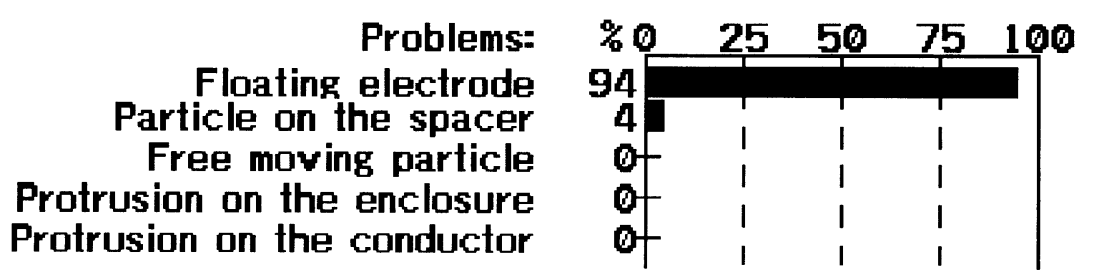

Figure 4. Frequency spectra and PD patterns measured. a, on 17 April 1998; b, on 25 June 1998; c, result of pattern recognition.

dates. At several points in time changes in the key value have been observed, as indicated in the figure.

As shown in Figure 3, there is an increase on 13 March 1998, indicated by point (1). Figure 4a shows the frequency spectrum measured on 13 March 1998. Some clear peaks can be observed which are the causes of this increase in the trend at point (1). The increase is introduced by a PD source, which has a PD pattern as shown next to the spectrum.

The trend shows a huge increase at 25 June 1998, indicated by (2) in Figure 3. Figure 4b shows the frequency spectrum measured 25 June 1998. Indeed, the amplitude of the frequency spectra is considerably increased compared with Figure 4a. Moreover, the PD pattern is completely different. After comparison of these measuring results with a reference database, the presence of an electrically floating part was concluded (see Figure 4c). This observation was confirmed by acoustic inspection and the exact location could be determined by this inspection as well. After repair of the electrically floating part, some changes in the trend have been observed as indicated by (3), (4) and (5) in Figure 3. These changes can be explained by adjustments made to the amplifiers. Furthermore, no changes in the frequency spectra and PD patterns have been observed.

\subsection{DISCRIMINATION BETWEEN INSULATION DEFECTS}

Typical frequency spectra obtained for different defects, Figure 2, show some differences. It can be seen that in case of an electrically floating electrode, the amplitude decreases with increasing frequency. In case of a free moving particle, it is difficult to state something besides that it is a rather dense spectrum. In case of fixed protrusions, the spectral content is lower. So, although the shape of frequency spectra depends on the type of defect, but also on the GIS design and used UHF couplers, a significant difference can be seen in the indicated low-frequency span and high-frequency span [5, 16]: the peaks in the spectrum from a protrusion fixed to the high-voltage conductor are higher in the low-frequency span compared with a protrusion fixed to the enclosure and for the highfrequency span it is the other way around.

This can be explained by a simplified way how electromagnetic waves are excited as shown in Figure 5. This figure just shows two examples but in general this explains that TEM modes (low frequency waves) are easier excited if the defect is located at the high-voltage conductor and higher order modes (higher frequency waves) if the defect is located at the enclosure [16]. 


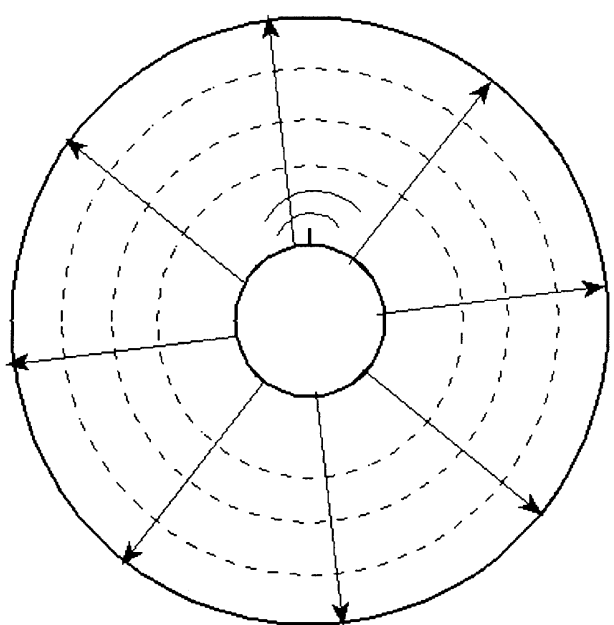

a)

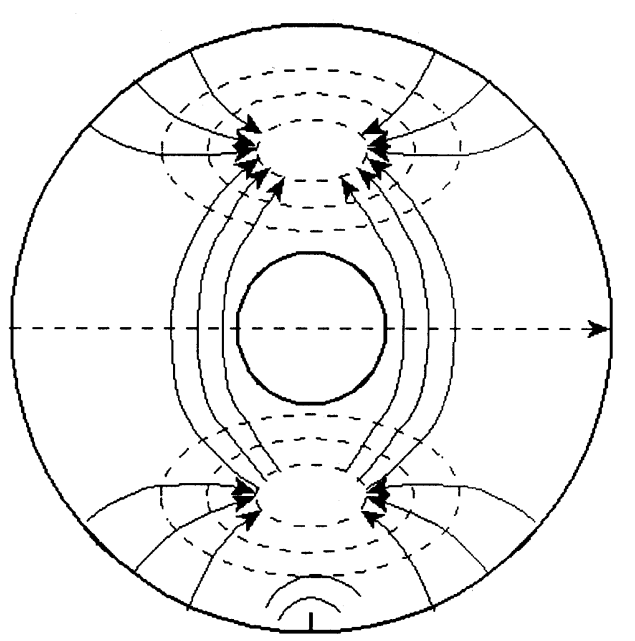

b)

Figure 5. Excitation of electromagnetic waves in case of a protrusion fixed to: a, the high-voltage conductor: easy excitement of TEM modes: lower frequencies; $b$, the enclosure: easy excitement of higher order modes: higher frequencies.

Figure 6 shows the comparison of the key value AR calculated for the low-frequency and high-frequency spans. Two different groups can be distinguished:

Group 1: the ratio between the UHF signal in the low frequency part and the UHF signal in the high frequency part is greater than 1 .

Group 2: the ratio between the UHF signal in the low frequency part and the UHF signal in the high frequency part is smaller than 1 .

It can be seen that the free moving particle and most of the protrusion fixed to the enclosure (LV protrusion) are in group 1 and the protrusion fixed to the high-voltage conductor (HV protrusion) and the electrically floating electrode in Group 2.

Moreover, it can be concluded that only analysis of the magnitude of two frequency spans is not sufficient to discriminate between defects as can be seen in case of the

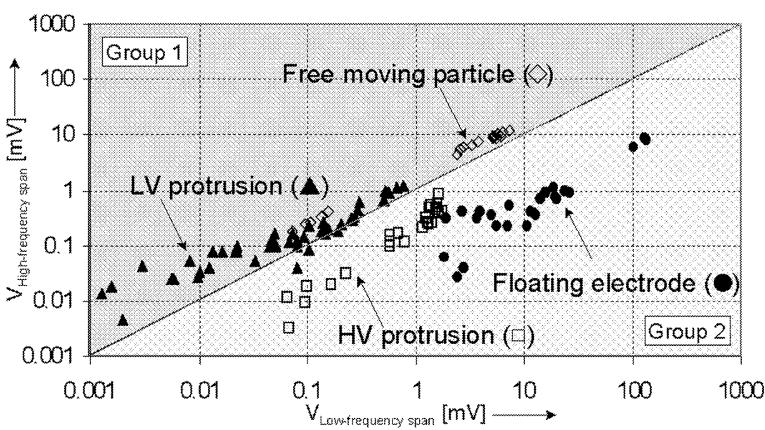

Figure 6. Comparing the key value AR in the low-frequency span $(100-475 \mathrm{MHz})$ and the high-frequency span $(850-1225 \mathrm{MHz})$ it is possible to distinguish two groups of frequency spectra: originating from defects on the high-voltage conductor or on the enclosure.

LV protrusion. Therefore additional analysis of frequency spectra using statistical analysis will be described in the following to improve this discrimination.

\section{STATISTICAL ANALYSIS OF FREQUENCY SPECTRA}

Clear differences can be observed in the shape of the frequency spectra obtained for four different defects, see Figure 2. This section describes whether it is possible to discriminate between different PD sources based on their representative frequency spectra, and uses statistical analysis for this purpose.

\subsection{DEFINITION OF STATISTICAL DISTRIBUTIONS}

Consider the frequency spectrum which consists of a series of amplitudes $S_{i}$, having a set of peaks $P K_{j}$. A peak is locally defined as $P K_{j}=S_{i}$ if

$$
\left(S_{i}>S_{i-1}\right) \wedge\left(S_{i}>S_{i+1}\right)
$$

The range of amplitudes of peaks in the frequency spectrum is divided into $\mathrm{N}$ windows, with a window size of

$$
\Delta A=\frac{P K_{\max }-P K_{\min }}{N}
$$

in which

$$
\left\{\begin{array}{l}
P K_{\max }=\left.\operatorname{MAX}\left(P K_{j}\right)\right|_{j=1} ^{m} \\
P K_{\min }=\left.\operatorname{MIN}\left(P K_{j}\right)\right|_{j=1} ^{m}
\end{array}\right.
$$

So now the following amplitude windows AW can be defined by

$$
\left\{\begin{array}{l}
A W_{1}=\left[P K_{\min }, P K_{\min }+\Delta A\right] \\
A W_{2}=\left[P K_{\min }+\Delta A, P K_{\min }+2 \cdot \Delta A\right] \\
\vdots \\
A W_{N}=\left[P K_{\min }+(N-1) \cdot \Delta A, P K_{\max }\right]
\end{array}\right.
$$


a)

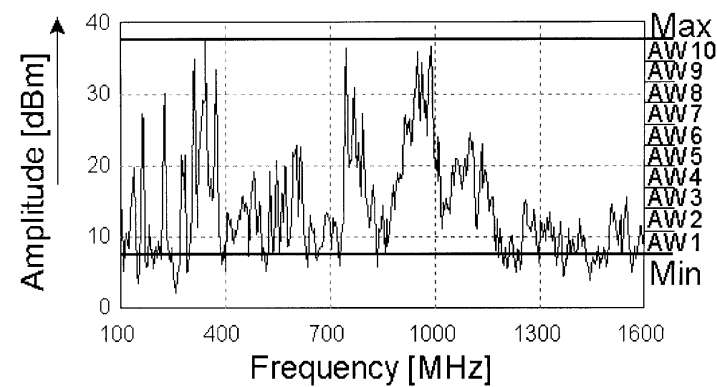

b)

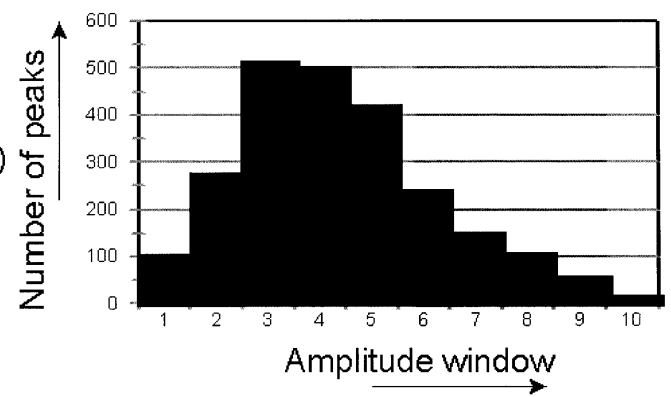

Figure 7. Amplitude window analysis. a, Division of the frequency spectrum into ten amplitude windows; $b$, The resulting amplitude distribution vs. amplitude-window number.

In figure $7 \mathrm{a}$ the division of the frequency spectrum into $N=10$ windows is shown; Figure $7 \mathrm{~b}$ shows the resulting distribution.

The above described method uses amplitude windows. A second way to derive statistical distributions comprises the following steps: 1) divide the frequency range of the frequency spectrum in certain frequency windows; 2) process the average amplitude in each frequency window; 3 ) apply the amplitude window method to the average amplitudes of each frequency window [17].

\subsection{SPECTRAL ANALYSIS OF EXPERIMENTAL RESULTS}

Figure 8 shows a comparison between the spectra of two defects: a free moving particle (Figure 8a) and a protrusion fixed to the HV conductor (Figure 8b). The statistical distributions based on the amplitude-window method and on the frequency-window method are shown in Figure $8 \mathrm{c}$ for a free moving particle and in Figure $8 \mathrm{~d}$ for the fixed protrusion.

Statistical moments, in particular the skewness and kurtosis, have been applied to these four distributions to analyse the shape of the distributions, Figures $8 \mathrm{e}$ and $8 \mathrm{f}$. A clear difference between both characterizations can be seen, which means that it is possible to discriminate between different spectra and therefore, in this case, between different defects.

After analysis of many frequency spectra it could be concluded that only these four quantities did not provide enough information to discriminate between the four typical defects shown in Figure 2. Therefore, the statistical moments were also applied to the low-frequency span and high-frequency span as indicated in Figure 2, and also the ratio of the amplitude in both spans as described in 3.3. As a result, the characterization of a frequency spectrum consists of thirteen values. Characterizations of the spectra of the four defects of Figure 2 (averaged over several measurements) are shown in Figure 9.

In this way, the feature vectors of 83 frequency spectra were processed and cluster analysis (using a Ward linkage method with an Euclidean distance measure) was applied to discriminate between defects. The result was the tree structure shown in Figure 10.

Cutting the tree at a certain level results in four main clusters. Analysis of these clusters shows that measurements of one type of defect are grouped in each cluster, except for cluster 4. Cluster 1 contains measurements of a protrusion fixed to the high-voltage conductor; cluster 2 contains measurements of a protrusion fixed to the enclosure and cluster 3 measurements of a free moving particle. Cluster 4 contains measurements from a free moving particle and an electrically floating electrode.

As indicated in Figure 10, two sub-clusters inside cluster 2 and 4 can be distinguished. Comparison of the spectra grouped in cluster $2 \mathrm{a}$ and $2 \mathrm{~b}$ did not provide any significant differences to explain the reason for both clusters. Both clusters found in cluster 4 can be explained: cluster $4 \mathrm{~b}$ represents the measurements obtained from an electrically floating electrode while cluster 4 a contains measurements obtained from a free moving particle. Further analysis of these measurements in cluster 4a showed that these were obtained at the very beginning of the PD activity coming from a free moving particle (shuffling particle). In this case very small contact noise-like PD activity was observed, which gives rather similar phase-resolved PD patterns as an electrically floating electrode, see Figure 11a, and differ completely from the typical PD patterns measured for moving and jumping particles, see Figures $11 \mathrm{~b}$ and $11 \mathrm{c}$.

\section{PD IDENTIFICATION}

As known, statistical analysis can be used to discriminate phase-resolved PD patterns [13]. The result can be visualized as a tree structure, like the one shown in Figure 10 . To clarify the result of statistical analysis only two features of the total feature vector are shown in Figure 12. In Figure 12a) two clusters can be defined. In one of these clusters, all the measurements obtained for a free moving particle are situated.

However, measurements obtained from protrusions fixed to the high-voltage conductor and from those fixed to the enclosure are mixed in the other cluster. So this method is not capable of discriminating between both types of protrusions. However, analysis of the PD patterns, which were clustered in each of these three clusters, shows three types of patterns, depending on the PD pro- 

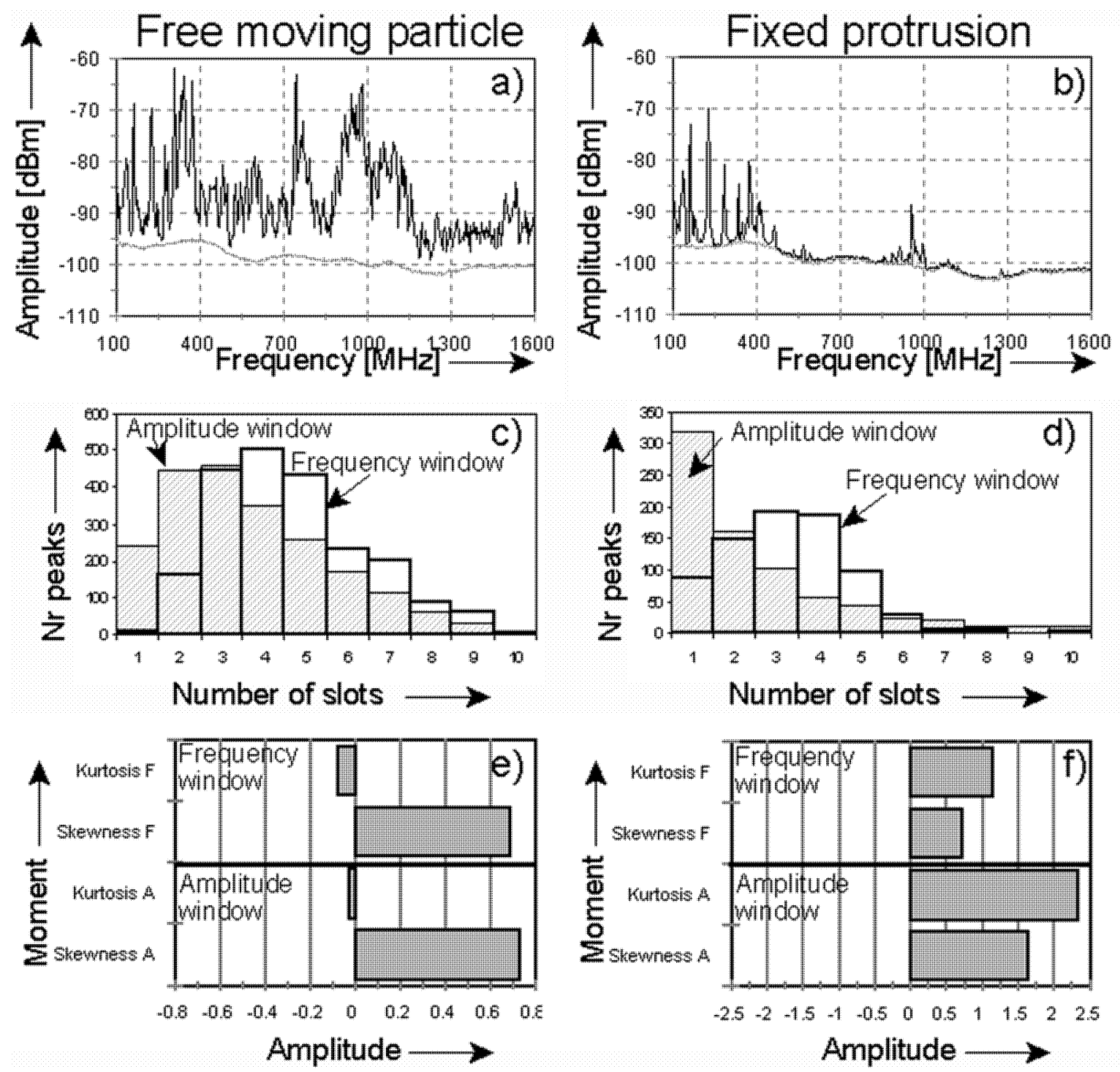

Figure 8. Example of statistical analysis of frequency spectra. a, Spectrum of a free moving particle; b, Spectrum of a protrusion fixed to the HV conductor; c, Distributions of a free moving particle; d, Distributions of a protrusion fixed to the HV conductor; e, Characterization of a free moving particle; $\mathrm{f}$, Characterization of a protrusion fixed to the HV conductor.

cess, see Figure 12c. It is known that for a protrusion fixed at the high-voltage conductor, the PD pattern at inception looks like pattern type 3 . Close to breakdown the pattern looks like pattern type 1 as shown in Figures $12 \mathrm{~b}$ and 12c. For a protrusion on the enclosure it is the other way around: around inception the PD pattern looks like pattern type 1 and near breakdown like pattern type 3 . Therefore it is not possible to determine whether the PD pattern is from a HV protrusion or LV protrusion. Only analysis of PD patterns is not sufficient to assess the risk for breakdown of fixed protrusions.

In addition to the identification technique using phaseresolved PD pattern analysis, also statistical analysis of the frequency spectra was used, see Figure 13. Three different clusters can be distinguished. Analysis of each cluster shows that each cluster only contains characterizations processed from frequency spectra of one type of defect: cluster 1 contains measurements from a protrusion fixed to the high-voltage conductor, cluster 2 from a protrusion fixed to the enclosure and cluster 3 from a free moving particle.

\section{ASSESSMENT OF RISK FOR BREAKDOWN}

According to the definition as given by the Society for Risk Analysis, risk analysis is an analytical process to provide information regarding undesirable events [17]. Risk assessment is the process of establishing information regarding acceptable levels of a risk of a defect to the insulation system [17]. Critical defects are those defects which are at the threshold of being harmful to the dielectric integrity of the GIS under normal ac operating stresses [2]. 

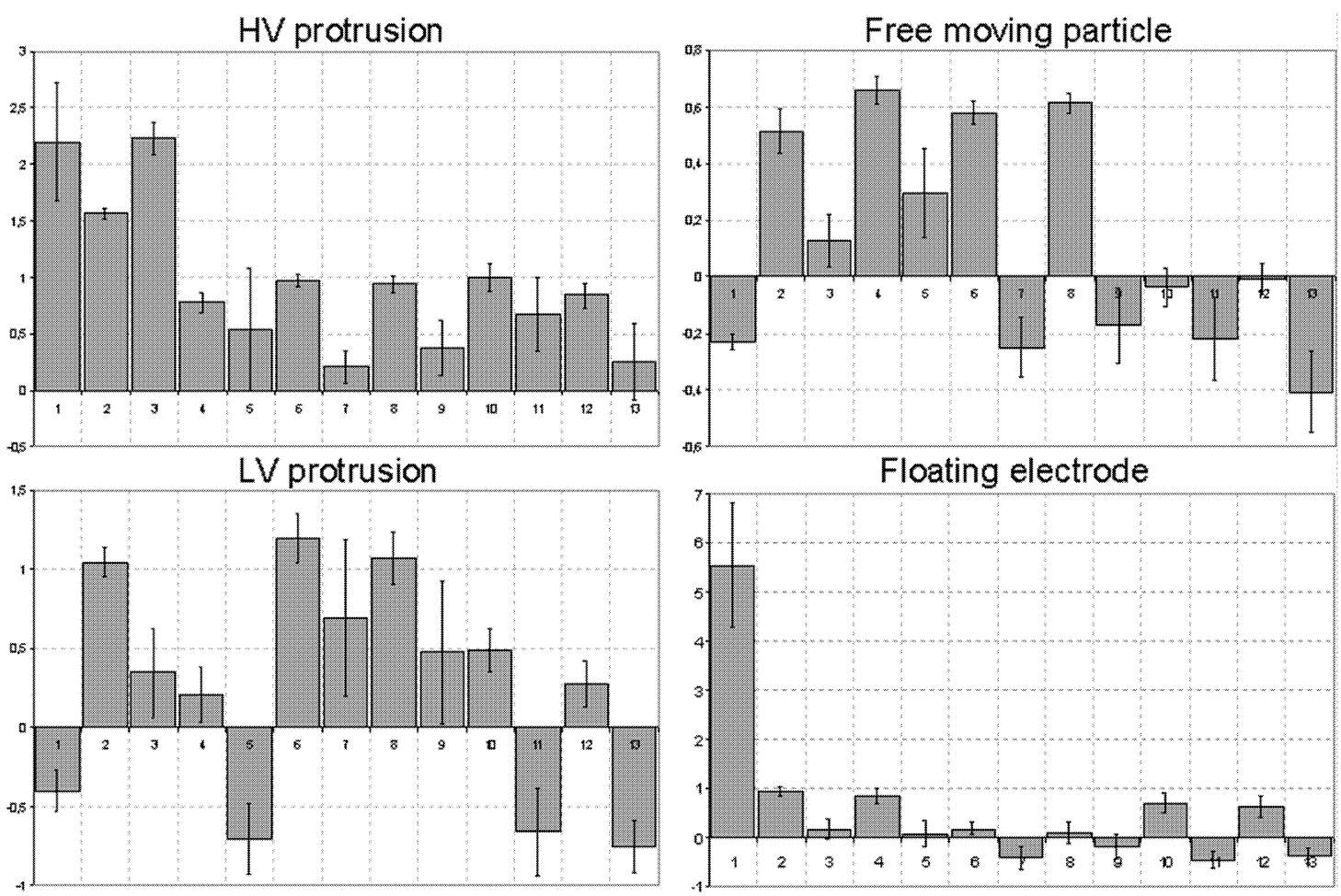

Figure 9. Feature vectors of the measured frequency spectra for different defects: HV protrusion, LV protrusion, free moving particle and electrically floating electrode.

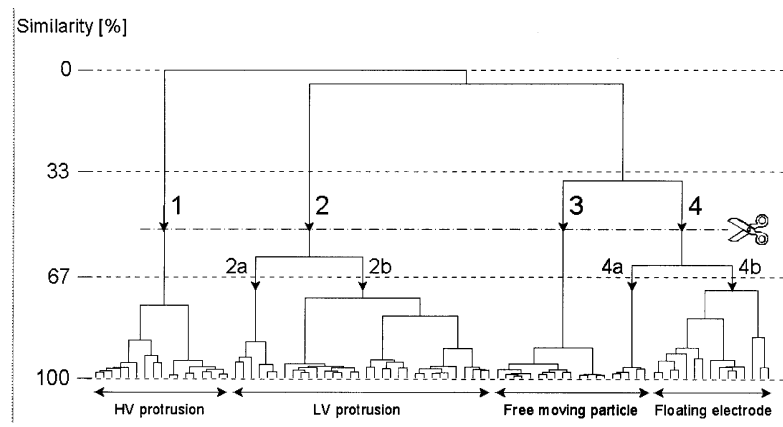

Figure 10. Cluster analysis applied to the feature vectors processed from the frequency spectra for four different defects: protrusion fixed to the high-voltage conductor (HV protrusion), protrusion fixed to the enclosure (LV protrusion), free moving particle and electrically floating electrode.

For undesirable events in the insulation system of $\mathrm{SF}_{6}$ gas-insulated systems an analysis process to assess the risk (called risk analysis process) involves the following four steps in condition assessment [4]:

- Detection.

- Identification.

- Location.

- Risk assessment of the defect.

In this section two examples of a first approach of the risk analysis process are described.
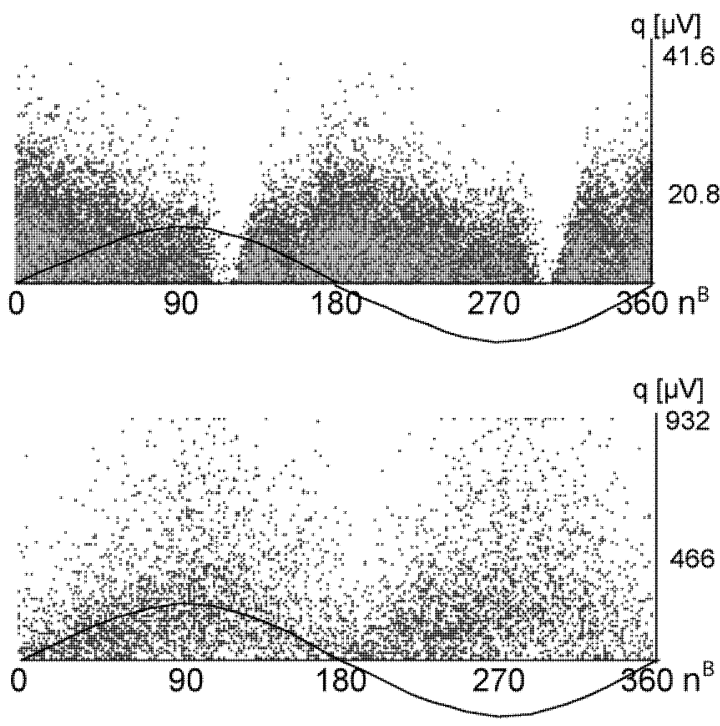

a)

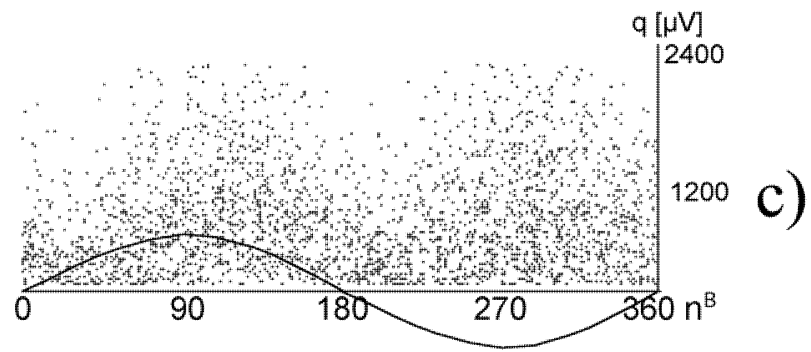

Figure 11. Typical phase-resolved PD patterns measured for a free moving particle when it is: a, shuffling; $b$, dancing; $c$, jumping. 

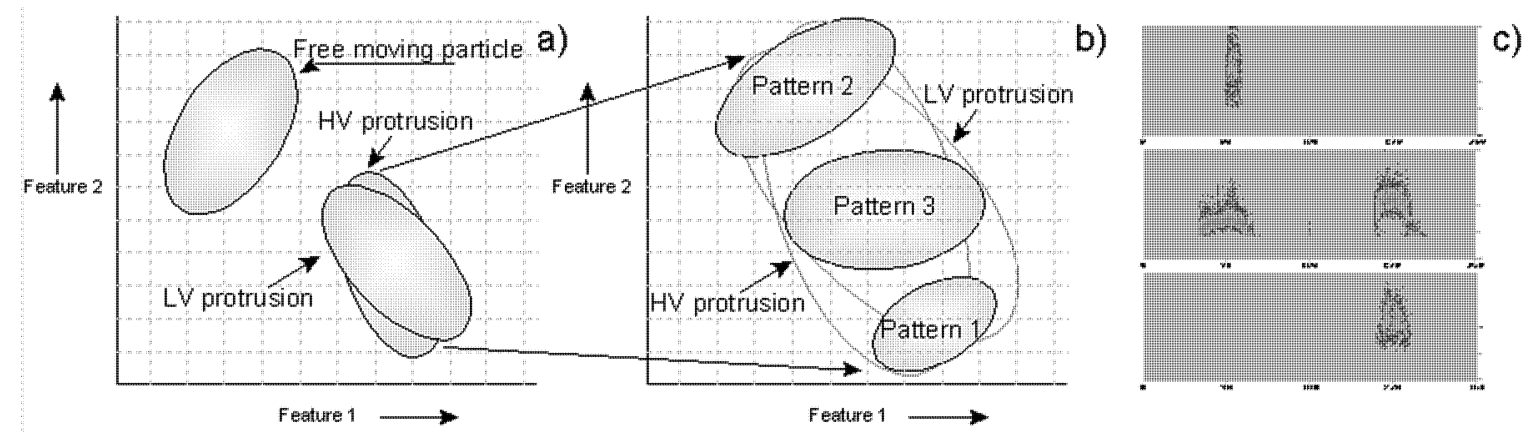

Figure 12. Cluster-formation after statistical analysis of phase-resolved PD patterns for: $a$, different defects; $b$, different patterns in case of fixed protrusions; c, the shape of the patterns in each cluster shown in Figure 12b.

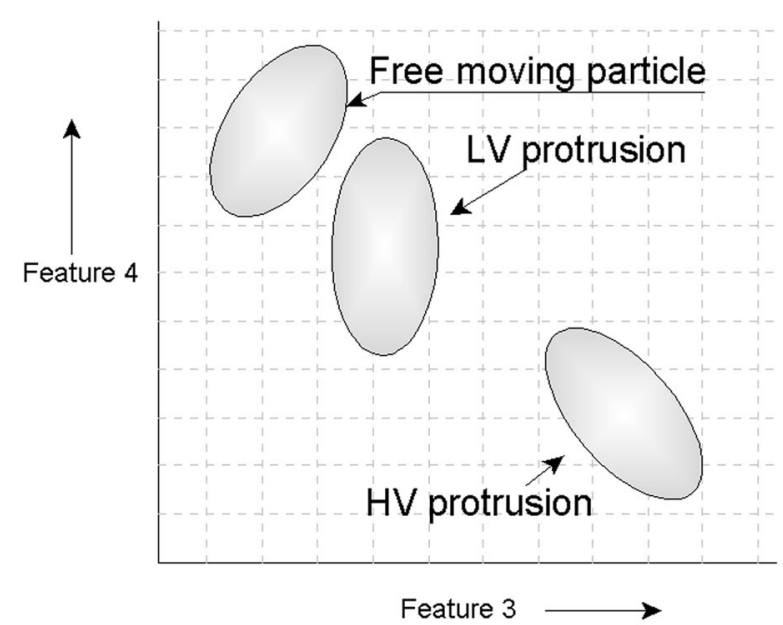

Figure 13. Cluster-formation after statistical analysis of frequency spectra for different defects: free moving particles, protrusions fixed to the high-voltage conductor (HV protrusion) and protrusions fixed to the enclosure (LV protrusion).

\subsection{FREE MOVING PARTICLES}

It is known that when a particle approaches the highvoltage conductor, a breakdown can occur [2]. Figure 11 shows examples of phase-resolved PD patterns obtained for three different movement stages of the free moving particle. The first movement stage is the shuffling particle. In this stage, the particle is not yet moving, but gives contact-noise-like discharges, which results in the PD patterns shown in Figure 11a.

The shape of the phase-resolved PD pattern changes as soon as the particle starts to move, see Figure 11b. This means that it is possible to distinguish between shuffling and moving particles using PD pattern analysis. However, except for a change in the maximum amplitude of the PD pulses, no change in the shape of the PD pattern can be observed if the particle starts to jump, see Figure 11c. This movement stage is the final stage close to breakdown. As a result, only analysis of phase-resolved PD patterns is not sufficient to determine the risk for a failure due to a free moving particle.

To improve the risk assessment of particles, the frequency spectra have been further analyzed. Examples of

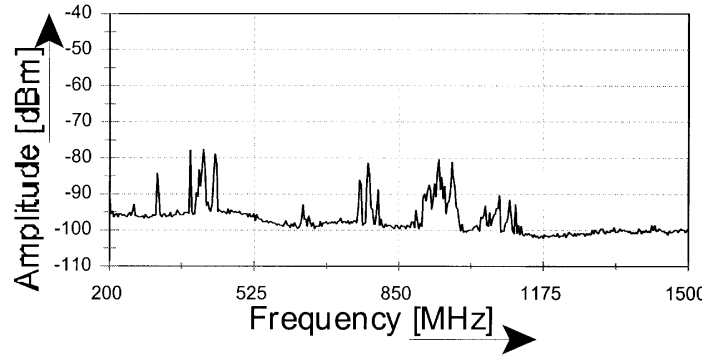

a)
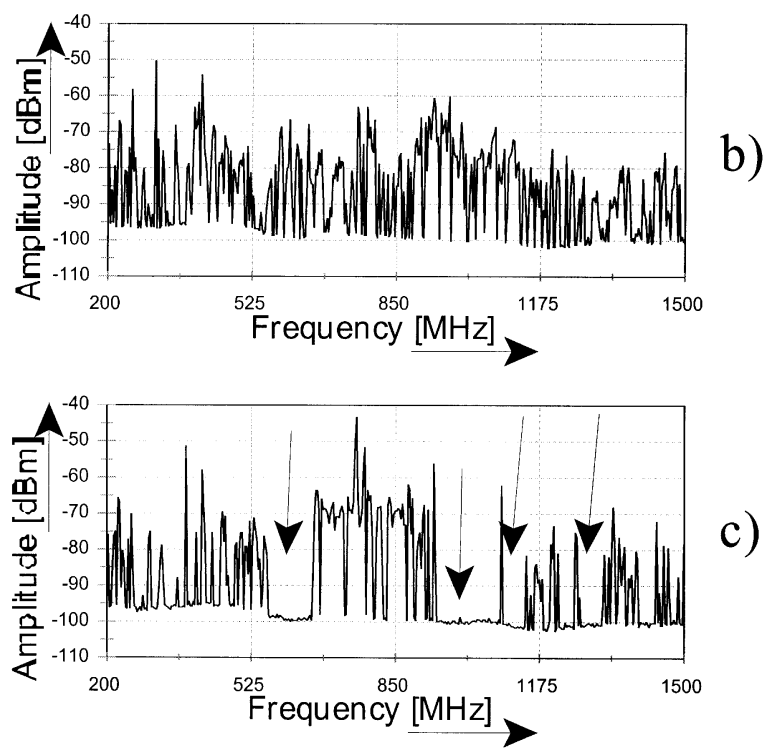

Figure 14. Examples of frequency spectra measured for different moving stages: a, shuffling particle; b, moving particle; $c$, jumping particle

frequency spectra are shown in Figure 14. As was the case with the PD patterns analysis, it is possible to discriminate between the shuffling stage and both other stages. However, in contrast to the phase-resolved patterns, there is a difference in the measured frequency spectra between the moving stage and the jumping stage. Empty parts or 'gaps' can be observed in the frequency spectrum for the jumping stage, which are indicated by arrows.

These gaps can be explained by the way the spectrum analyzer captures a frequency spectrum. A spectrum analyzer effectively performs a Fourier analysis of a (continu- 
ous) input signal. Like a radio receiver it tunes at consecutive frequencies and captures the amplitude of the spectral content of the input signal at the tuned frequency. It takes a certain amount of time for the SA to tune at all frequencies in the frequency spectrum, called sweep time. During the sweep time, the discharging signal at the input of the SA changes with each discharge. Therefore, the final captured frequency spectrum is built from several discharge signals.

If the particle is jumping, there is no discharge activity and therefore no input signal. This results in the empty part of the frequency spectrum. So the empty parts occurring in a frequency spectrum have nothing to do with no spectral content of the discharging signal. Only due to the minimal amount of time required to capture a frequency spectrum, these empty parts appear as soon as the particle is jumping and, for some time, no discharge activity occurs. Because the particle movement is a random process, the empty parts in the spectra can change from sweep to sweep.

To discriminate between moving and jumping particles, the gaps in the frequency spectra are used. One spectral measurement consists of 20 single sweeps with a sweep time of $5 \mathrm{~s}$. If the particle is moving, no gaps will occur, and if the particle is jumping, these gaps will occur at random frequencies. Therefore, two new frequency spectra are post-processed from one spectral measurement: an averaged spectrum and a spectrum consisting of the maximum amplitude at each frequency taken from all sweeps. Table 1 shows the results of calculating the amplitude of both spectra for both movement stages.

From Table 1 can be concluded that the magnitude of the hold-max spectrum is larger compared to the averaged one. Moreover, looking at the hold-max spectrum, the magnitude increases once the particle starts jumping. This can be explained by the fact that the charge acquired by a particle depends linearly on the applied field strength [18]. In order to go from the moving to the jumping stage, the field strength was increased and as a result, the magnitude of frequency spectrum increases too. In contrast, the magnitude of the averaged spectrum decreases once the particle starts jumping. This can be explained by the gaps in the frequency spectrum, which reduces the final amplitude if an averaging technique is used over the 20 acquired sweeps.

Because of the gaps in the spectra for jumping particles, it can be expected that the ratio of the hold-max spectrum and the averaged spectrum is bigger for jumping

Table 1. Key-value AR calculated from the post-processed frequency spectra of a moving and jumping particle, using an averaging or hold-max processing technique.

\begin{tabular}{cccc}
\hline Movement & Averaged spectrum & Hold-max spectrum & Ratio \\
\hline Moving & $6.3 \mathrm{mV}$ & $22.6 \mathrm{mV}$ & 3.6 \\
Jumping & $5.8 \mathrm{mV}$ & $28.8 \mathrm{mV}$ & 5.0 \\
\hline
\end{tabular}

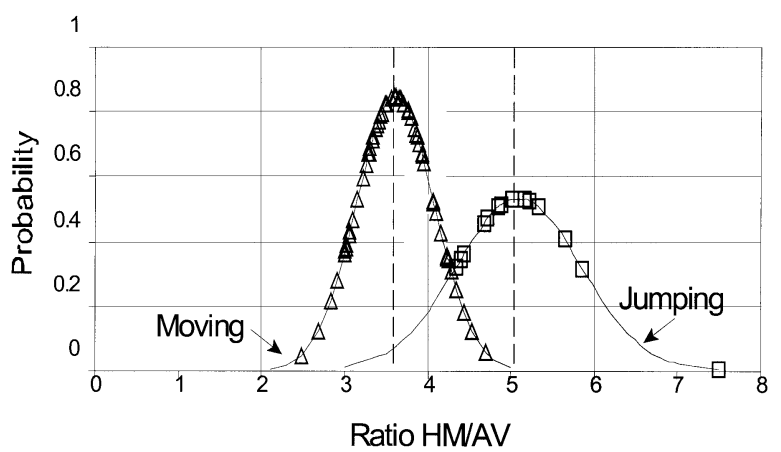

Figure 15. Probability density distribution for moving and jumping particles. $\Delta$ Measured points moving particles; $\square$ Measured points jumping particles.

particles compared to moving particles. For further analysis, this aspect has been used. For a general approach, the ratio of values has been used, as can be seen in the fourth column of Table 1 . Therefore the ratios of about 80 measurements have been processed and analysed. This resulted in the probability density distributions for the measured ratios, see Figure 15. It is clear that the ratio of moving and jumping particles are differently distributed.

Above shown distributions have been used to estimate the movement stage of particles, located in other GIS equipment or with different particle dimensions etc. In total 18 particles were analysed.

For 13 particles (numbered 1-13) it was known that they were only moving inside the GIS and not jumping. From the obtained frequency spectra, the ratio between the hold-max and averaged spectrum was calculated and compared with the probability distributions. As a result, Figure 16 shows for each investigated particle, the probability for the movement stage: moving (hollow bars) or jumping (filled bars). To determine the particle movement, a

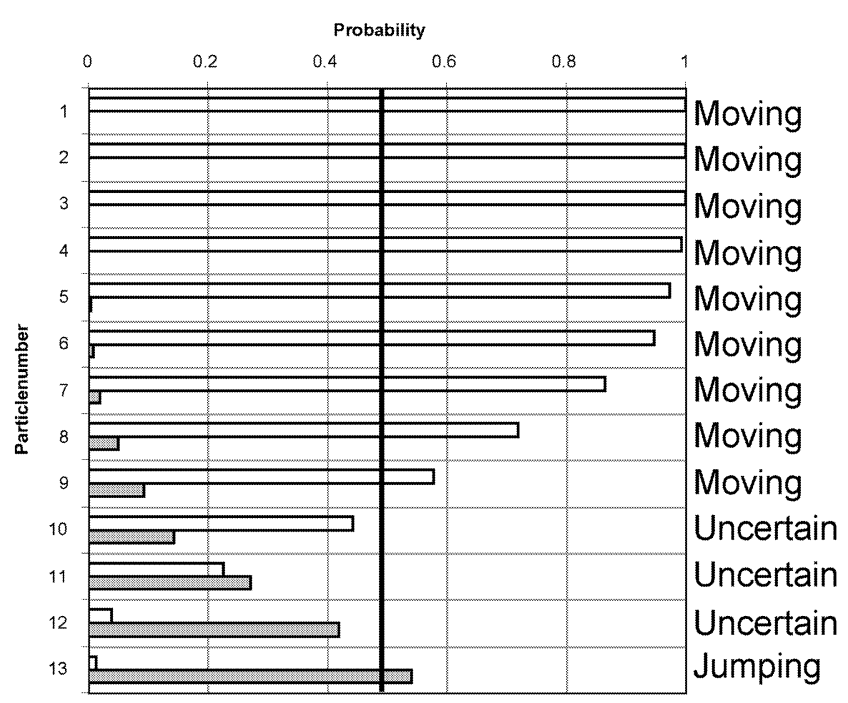

Figure 16. Probability analysis of moving particles: the hollow bars indicate the probability that the particle is moving; the filled bars indicate the probability that the particle is jumping. 


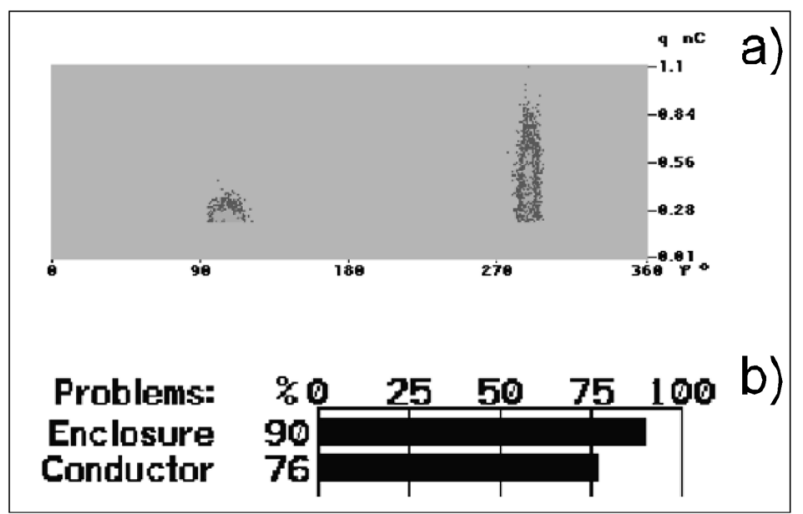

Figure 17. a, Phase-resolved PD pattern from a fixed protrusion; $b$, the statistical defect recognition based on phase-resolved PD pattern analysis.

threshold of $50 \%$ probability has been used. Next to the graph, the estimated particle movement is written. As can be seen, particles 1 to 9 have a probability larger than $50 \%$ that it is moving. Only particle 13 has a probability larger than $50 \%$ that it is jumping. Particles $10-12$ have a probability below $50 \%$ for both moving and jumping and the classification is uncertain. So from the thirteen investigated moving particles, one was incorrectly classified as a jumping particle and the movement of three other particles was uncertain.

\subsection{FIXED PROTRUSIONS}

Figure17a shows a phase-resolved PD pattern, which has been identified as a fixed protrusion, Figure 17b. For this purpose the measured PD pattern was compared with the

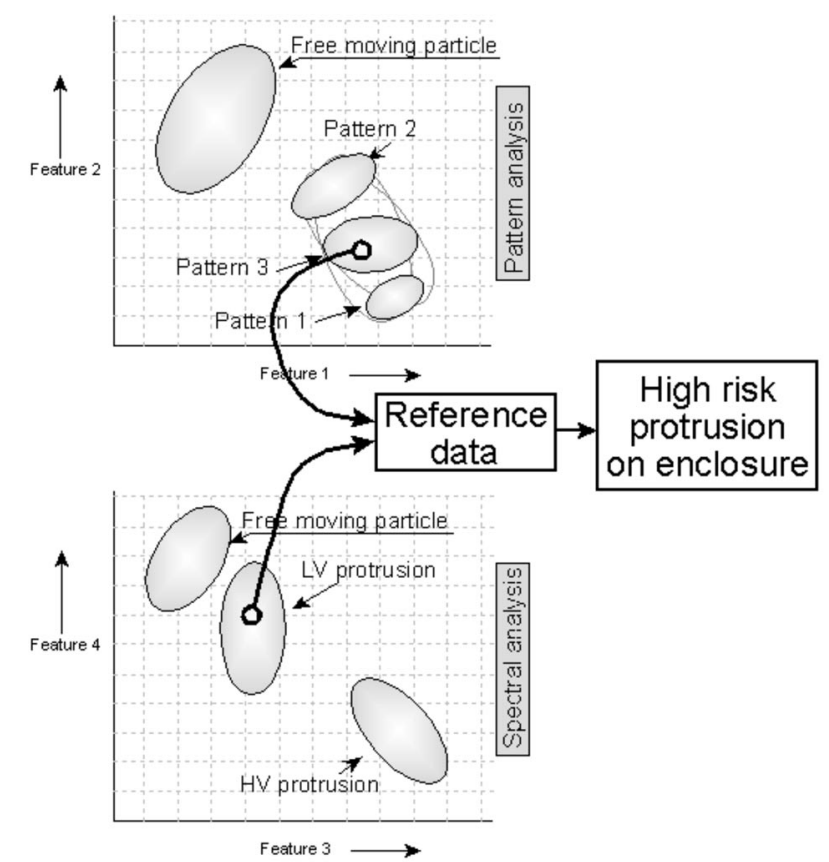

Figure 18. Assessment of the risk for breakdown of fixed protrusions.
PD patterns stored in a reference database, in which different types of protrusions are stored, represented by several types of PD patterns. As expected nothing can be concluded about the location of this protrusion: fixed to the high-voltage conductor or to the enclosure.

Above-mentioned defect was further analysed by combined PD pattern analysis and spectral analysis, see Figure 18. From the PD pattern analysis can be concluded that the pattern is originating from a fixed protrusion, giving partial discharge activity with pattern type 3 . From spectral analysis it can be concluded that the protrusion is fixed to the enclosure. Comparing this with knowledge stored in an expert system and as explained in Section 5, a high-risk (close to breakdown) protrusion fixed to the enclosure could be concluded.

\section{CONCLUSIONS}

1. Spectral key-values can be used for trend-analysis of PD activity in GIS and to discriminate between two groups of insulation defects.

2. Statistical analysis of peak amplitude distributions processed from frequency spectra can be used to distinguish between different insulation defects.

3. To assess the risk of fixed protrusions for ac breakdown, a combined analysis of frequency spectra and phase-resolved PD patterns is necessary.

4. To discriminate between moving and jumping particles spectral analysis can be used. The most critical stage in the particle movement is jumping and can be recognised using probability distributions processed from spectral analysis.

\section{ACKNOWLEDGMENT}

The authors acknowledge Alstom T\&D in France and TenneT in The Netherlands for their support.

\section{REFERENCES}

[1] T. M. Chan, F. Heil, D. Kopejtkova, P. O'Connell, J. P. Taillebois and I. Welch, "Report on the second international survey on high voltage gas insulated substations (GIS) service experience", CIGRE, paper 23-102, 1998.

[2] CIGRE WG 15.03, "Diagnostic Methods for GIS Insulating Systems", CIGRE, paper 15/23-01, 1992.

[3] B. F. Hampton, J. S. Pearson, C. J. Jones, T. Irwin, I. M. Welch and B. M. Pryor, "Experience and Progress with UHF Diagnostics in GIS", CIGRE, paper 15/23-03, 1992.

[4] L. E. Lundgaard, B. Skyberg, A. Diessner and A. Schei, "Method and Instrumentation for Acoustic Diagnoses of GIS", CIGREreport 15-309, Paris, 2000.

[5] H. D. Schlemper, R. Kurrer and K. Feser, "Sensitivity of on-site partial discharge detection in GIS", Proc. $8^{\text {th }}$ Int. Symp. HV Engineering, Yokohama, Vol. 3, pp. 157-160, 1993.

[6] B. F. Hampton and R. J. Meats, "Diagnostic measurements at UHF in gas insulated substations", IEE Proc., Vol. 135, Pt. C, pp. $137-144,1988$.

[7] J. S. Pearson, B. F. Hampton and A. G. Sellars, "A continuous UHF monitor for gas-insulated substations", IEEE Trans. Electr. Insul., Vol. 26, pp. 469-478, 1991. 
[8] IEC Publication 60270, Partial Discharge Measurements, 3rd Edition, 2000.

[9] CIGRE WG 33/23.12, "Insulation Co-ordination of GIS: return of experience, on site tests and diagnostic techniques", Electra No. 176, pp. 67-97, 1998.

[10] CIGRE TF 15/33.03.05, "PD Detection System for GIS: Sensitivity Verification for the UHF Method and the Acoustic Method", Electra No. 183, pp. 75-87, 1999.

[11] S. Meijer, E. Gulski, J. J. Smit, F. J. Wester, Th. Grun and M. Turner, "Interpretation of PD in GIS using Spectral Analysis", 11th ISH, London, UK, Vol. 5, pp. 124-127, 1999.

[12] E. Gulski, S. Meijer, W.R. Rutgers and R. Brooks, "Recognition of $\mathrm{PD}$ in $\mathrm{SF}_{6}$ insulation using digital data processing", IEEE Conf. Electr. Insul. Dielectr. Phenomena, pp. 577-580, 1996.

[13] E. Gulski, "Digital analysis of partial discharges", IEEE Trans. Dielectr. Electr. Insul., Vol. 2, pp. 822-837, 1995.

[14] S. Meijer, E. Gulski and J. J. Smit, "Pattern Analysis of Partial Discharges in $\mathrm{SF}_{6}$ ", IEEE Trans. Dielectr. Electr. Insul., Vol. 5, pp. $830-842,1998$.

[15] W. Leon and L.W. Couch II, Digital and Analog Communication Systems, Macmillan Publishing Company, New York, 1993.

[16] T. Tsurimoto, H. Moto, M. Doi, K. Ito and H. Fujii, "Correlation between two frequency components of various PD pulses in GIS”, ISEIM, P2-15, pp. 653-656, 1998.

[17] S. Meijer, "Partial Discharge Diagnosis of High-Voltage GasInsulated Systems", Optima Grafische Communicatie Rotterdam, ISBN 90-77017-23-2, 2001.

[18] M. Holmberg, "Motion of Metallic Particles in Gas Insulated Systems", Chalmers Bibliotek, Reproservice, Göteborg, ISBN 91-7197-533-0, 1997.

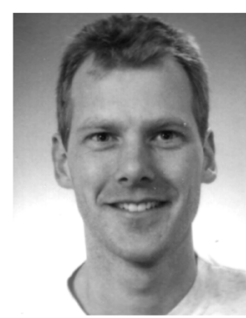

Sander Meijer was born in 1971 in The Netherlands. He received the Master degree in 1995 and the Ph.D. degree in 2001 for his research in the field of the ultra-high frequency partial discharge diagnostics. At present, he is an Assistant Professor in the Department of High Voltage Technology and Management and is involved in education and research in the field of high frequency measuring techniques for insulation diagnosis of high voltage components. He is active in different Cigréorking groups and task forces.

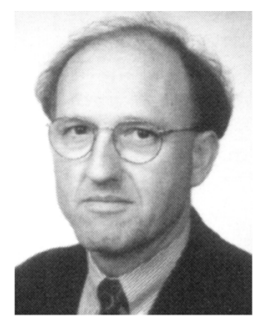

Johan J. Smit was born in 1949 in the Netherlands. He has been a Professor of High Voltage Technology at the Delft University of Technology since 1996 . Currently his specific areas of interest are HV materials, advanced diagnostics and asset management systems. He is presently also a member of the supervisory board of $\mathrm{TZH}$, the Power Grid Company of South Holland. After receiving the Master degree in 1974 on experimental physics at the University of Amsterdam he received the Ph.D. degree from the State University of Leiden in 1979 for his research on magnetism on behalf of the National Science Foundation. Next he was employed for 17 years at KEMA's testing, consultancy and engineering company, Arnhem, as Section Manager of Transmission and Distribution. He is a member of the Technical Committee of IEC 98 on Electrical Insulation Systems. From 1993 he has been Secretary of CIGRE Study Committee 15, Materials for Electrotechnology, and since 1999 its chair. 